\title{
Article
}

\section{Face morphology: Can it tell us something about body weight and fat?}

\author{
Pascali, MA, Giorgi, D, Bastiani, L, Buzzigoli, E, Henriquez, P, \\ Matuszewski, BJ, Morales, MA and Colantonio, S \\ Available at http://clok.uclan.ac.uk/15388/
}

Pascali, MA, Giorgi, D, Bastiani, L, Buzzigoli, E, Henriquez, P ORCID: 00000001-6582-5351, Matuszewski, BJ ORCID: 0000-0001-7195-2509, Morales, MA and Colantonio, S (2016) Face morphology: Can it tell us something about body weight and fat? Computers in Biology and Medicine, 76 . pp. 238-249. ISSN 0010-4825

It is advisable to refer to the publisher's version if you intend to cite from the work. http://dx.doi.org/10.1016/j.compbiomed.2016.06.006

For more information about UCLan's research in this area go to http://www.uclan.ac.uk/researchgroups/ and search for < name of research Group $>$.

For information about Research generally at UCLan please go to http://www.uclan.ac.uk/research/

All outputs in CLoK are protected by Intellectual Property Rights law, including Copyright law. Copyright, IPR and Moral Rights for the works on this site are retained by the individual authors and/or other copyright owners. Terms and conditions for use of this material are defined in the policies page.

\section{CLoK}

Central Lancashire online Knowledge www.clok.uclan.ac.uk

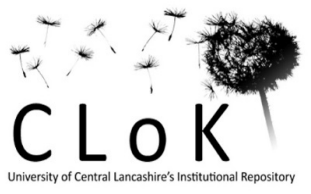




\title{
Face morphology: can it tell us something about body weight and fat?
}

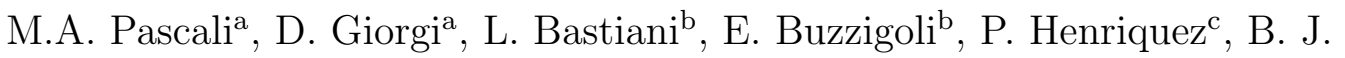

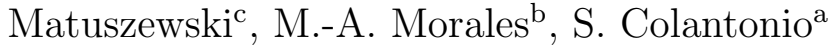 \\ ${ }^{a}$ Institute of Science and Information Technology, National Research Council, Pisa, Italy \\ ${ }^{b}$ Institute of Clinical Physiology, National Research Council, Pisa, Italy \\ ${ }^{c}$ School of Computing Engineering and Physical Sciences, University of Central \\ Lancashire, Preston, UK
}

\begin{abstract}
This paper proposes a method for an automatic extraction of geometric features, related to weight parameters, from 3D facial data acquired with lowcost depth scanners. The novelty of the method relies both on the processing of the 3D facial data and on the definition of the geometric features which are conceptually simple, robust against noise and pose estimation errors, computationally efficient, invariant with respect to rotation, translation, and scale changes. Experimental results show that these measurements are highly correlated with weight, BMI, and neck circumference, and well correlated with waist and hip circumference, which are markers of central obesity.

Therefore the proposed method strongly supports the development of interactive, non obtrusive systems able to provide a support for the detection of weight-related problems.
\end{abstract}

Keywords: Facial morphology, 3D scanning \& reconstruction, Weight \& fat-related parameters, BMI, Structural Equation Model

\section{Introduction}

Overweight and obesity (defined as abnormal or excessive fat accumulation) represent a major risk factor for a large spectrum of diseases, including cardiovascular disease, diabetes [1,2], musculoskeletal disorders, and some cancers (endometrial, breast, and colon). Data published by the Global Health Observatory in [3] show that excess body fat affects a large part of the adult population (aged 18 and over): around 39\% were overweight in 2014 
and $36.1 \%$ in 2010 ; while obese adult people were $13 \%$ in 2014 , and $11.3 \%$ in 2010. Hence, overweight and obesity are increasing, and cause direct and indirect costs, stressing healthcare and social resources: strong actions to prevent unhealthy behaviours in the general population are mandatory.

In recent years, personal health monitoring systems are gaining popularity, since individuals are increasingly more motivated to play an active role and are shifting from passive recipients of care towards actively managing their own health [4]. The SEMEOTICONS project (described also in $[5,6]$ ) falls in this domain: it aims to build a sensorized platform, the Wize Mirror, able to track over time the individual health status, and offer a tailored guidance towards lifestyle improvements, as described in $[7,6]$. Such a mirror arises from the integration of different modules, processing different data, which are acquired unobtrusively. In this paper, the advances in 3D scanning technologies and 3D geometry analysis are exploited to develop a method for the automatic and reliable estimation of weight-related health parameters, as a key component of easy-to-use, low-cost yet accurate healthcare tools. In the Wize Mirror, the estimation of such parameters will be complemented with results from the analysis of skin composition, emotional status, heart rate, endothelial functionality, chemical composition of the exhaled, in order to draw an overall picture of the individual's wellbeing status with respect to the cardio-metabolic risk.

The peculiar focus of this work is on the ability of digital measurements on 3D face scans to enclose the information about body weight and fat, which is measured in literature through well established indicators, such as weight, Body Mass Index (BMI), waist circumference, hip circumference and neck circumference. We focus on the face, following the principles of medical semeiotics, which considers the face as a mirror of wellbeing $[8,9,10]$. The challenge is significant: though it is well known that the face is involved in the process of fat accumulation, there is no consensus in the literature about which are the facial morphological correlates of body weight and related indexes (Section 2.2).

The contribution of this work is three-fold, on both the technological and the clinical side:

- We propose a low-cost, yet accurate, 3D face reconstruction system (Section 3.1); this makes the proposed method a viable solution in everyday scenarios, including home, pharmacies, and schools.

- We define a set of automatic digital face measurements based on mod- 
ern 3D shape analysis techniques (Section 3.2), which can be accurately computed without requiring any manual intervention.

- We show evidence of the correlation of the proposed facial measurements with standard parameters of overweight and obesity (including weight, BMI, waist circumference, hip circumference, neck circumference, and fat mass) in a study on 30 volunteers (Section 4); this evidence may support the adoption of the proposed measurements in self-monitoring applications for wellbeing.

\section{State of the art}

Anthropometry is the discipline which deals with the study of body and face morphology: it has been used for decades to measure individuals and quantify human physical variation through measurements performed manually by trained personnel with results often affected by inter- and intraobserver variability. Section 2.1 reports on the most common parameters to estimate the size and composition of the human body, and also to assess overweight and obesity.

Recently, the development of 3D scanning technologies opened a new field of research named digital anthropometry. The accuracy of digital measurements has been shown compatible with applications in forensic medicine, ergonomics, clothing industry, and medical settings [11, 12]. In Section 2.2, we report on existing computational measures related to overweight, obesity and cardio-metabolic risk.

\subsection{Standard anthropometric measurements}

Anthropometry, in the medical domain, refers to a set of simple, inexpensive and non-invasive methods to understand the physical properties of the human body and face, such as size and shape. Also, measurements and indexes derived from anthropometry may be used to monitor the nutritional status, and are currently studied to disclose existing relations with syndromes, as the obstructive sleep apnea, reported by Balabilh et al. in [13]), and health risk factors, such as the cardiovascular or the cardio-metabolic risk, in [14] by Millar et al.

The first indicator of generalized obesity, probably the most used and debated, is the body mass index (BMI), introduced in [15]. BMI is computed using only weight and height $[\mathrm{BMI}=$ (weight in kilograms)/(height in 
meters $)^{2}$; although simple, it shows a high correlation (0.7 to 0.8) with body fat in the adult general population, as reported in $[16,17]$. Hence, BMI is still used for statistical surveys of large population. Nonetheless its cut-off values are the same for male and female subjects, for all ages in the adult population (18 years and over). On the contrary, it is a crude measure, not distinguishing lean mass from fat. Scholars continue working on new measures and indexes, superior to BMI for the prediction of the overall health status.

Among the measures proposed to predict the cardio-metabolic risk we mention the following:

- the waist circumference (WC), a relatively simple and convenient measure to detect central fat accumulation [18, 19, 20];

- the hip circumference (HC), which provides additional information about the hip region, negatively associated with health outcomes in women [21];

- the waist-to-hip ratio (WHR), as it indicates increased cardiovascular risk [22];

- the neck circumference (NC), being a marker of central obesity, and associated with many fat-related anthropometric measurements and cardiovascular risk factor $[23,24,25]$.

In the present study weight, $\mathrm{BMI}, \mathrm{WC}, \mathrm{HC}$, and $\mathrm{NC}$ are considered as reference anthropometric measurements against which to validate the digital face measurements defined in Section 3.2.

\subsection{Digital anthropometric measurements}

State-of-the-art shape analysis provides a rich and powerful set of tools which automatically extract information from a 3D object (see [26] for a recent review). Computer vision and computer graphics have been applied to body analysis to estimate height, weight and other parameters enclosed in the body appearance.

Velardo and Dugelay presented a regression model for the weight estimation based on a set of geometric body measurements extracted from the 2D body silhouette [27]. They also propose in [28] an automatic vision-based system for estimating the subjects' absolute weight from a frontal 3D view of 
the user, acquired through a low-cost depth sensor; example of applications of such a system are extreme environments and circumstances in which a standard scale cannot work or cannot be used.

Giachetti at al. [29] used heterogeneous body scans as input data for the automatic extraction of geometrical parameters related to body fat. Their aim was the computation of parameters not dependent on the precise location of anatomical landmarks, and robust against pose and mesh quality, so as to be used in healthcare applications. They found that several parameters were highly correlated with total-body-less-head fat and trunk fat (computed via Dual-Energy X-rays Absorptiometry scanning).

As far as faces are concerned, most of the methods proposed in the literature are based on 2D images. Moreover, no conclusive results can be drawn about the morphological facial correlates of body fat. In [30] Ferrario et al. observed an increase in some facial dimensions in a study on the face morphology of obese adolescents. Djordjevic et al. in [31] reported an analysis of facial morphology of a large population of adolescents under the influence of confounding variables: though the statistical univariate analysis showed that four principal face components (face height, asymmetry of the nasal tip and columella basis, asymmetry of the nasal bridge, depth of the upper eyelids) correlated with insulin levels, the regression coefficients were weak, and no significance persisted in the multivariate analysis.

Only very few methods exploit 3D information: one of the most interesting is presented by Banabilh et al. in [13]), in which the craniofacial obesity, assessed via 3D stereo-photogrammetry, is correlated with the obstructive sleep apnea syndrome.

In adults, Lee et al. proposed in [32] a prediction method of normal and overweight females based on BMI using geometrical facial features only. The features, measured on 2D images, include Euclidean distances, angles and face areas defined by selected soft-tissue landmarks. The study was extended and completed in [33] by investigating the association of visceral obesity with facial characteristics, so as to determine the best predictor of normal waist and visceral obesity among the considered facial characteristics. Cross-sectional data were obtained from a population of over 11 thousand adult Korean men and women aged between 18 and 80 years. Also, in [34] Giorgi et al. defined a more complex shape descriptor from the geometric theory of persistent homology, to analyse the face morphology encoded in a set of 23 landmarks (a subset of the facial Farkas landmarks, defined in [35]), and tested it on a synthetic dataset of 3D faces. 
The present study differs from the state of the art in some aspects: the method proposed for the self-assessment of the wellbeing status with respect to the cardio-metabolic risk is fully automatic, in order to be integrated in a more complex system; also, it processes 3D face data acquired with a lowcost depth sensor. In spite of the low-cost of the sensor used, and of the low computational cost of the implemented methods, in Section 4, results showed that a relevant relation may exist among face morphology and body weight and fat-related parameters, including markers of central obesity, The present study differs from the state of the art in some aspects: the method proposed for the self-assessment of the wellbeing status with respect to the cardiometabolic risk is fully automatic, in order to be integrated in a more complex system; also, it processes 3D face data acquired with a low-cost depth sensor. In spite of the low-cost of the sensor used, and of the low computational cost of the implemented methods, in Section 4, results showed that a relevant relation may exist among face morphology and body weight and fat-related parameters, including markers of central obesity, an important component of cardio-metabolic risk.

\section{Methods}

The proposed study is based on two main components: the low-cost 3D reconstruction system of faces, described in Section 3.1, and the computation of geometrical measurements on the 3D facial data, in Section 3.2.

\subsection{Low-cost $3 D$ reconstruction}

This section describes an inexpensive system for 3D geometric face reconstruction based on readily available depth sensors such as Kinect or Asus Xtion. The system acquires and process the data in order to recover a good $3 \mathrm{D}$ reconstruction of faces: a manifold mesh with one boundary component, accurate enough for the extraction of informative facial features.

The proposed 3D reconstruction method recovers point clouds from a set of depth frames and fuses them into a single global surface. This is achieved using a structure from motion algorithm [36]. The data fusion is performed by tracking the global model using a coarse-to-fine iterative closest point (ICP) algorithm and the surface reconstruction is estimated using a truncated signed distance function (TSDF) and a Poisson meshing algorithm [37].

Before applying the reconstruction procedure, a face segmentation stage has to be performed on the depth frames in order to extract only the infor- 
mation representing the face from the depth data. This step eliminates background objects, body parts or hair from the reconstruction process. Without the segmentation the reconstruction can be noisy or/and heavily distorted. Additionally, this stage needs as a first step the face pose estimation in order to be able to select the region of interest from the depth frames.

\subsubsection{Face pose estimation}

The proposed face pose estimation consists of calculating the head position in $3 \mathrm{D}$ space $(\mathrm{x}, \mathrm{y}, \mathrm{z})$ and the orientation expressed by the three Euler angles (pitch, yaw, roll). The 3D pose of the face is calculated using the technique developed in [38], which is based on a random forest face pose estimation method [39] and a Kalman filter tracking. The Kalman filter is utilised to perform head pose tracking, by filtering the measurements provided by the face pose estimator. Moreover, using this approach, outliers can be detected and the missing measurements effectively handled.

\subsubsection{Face segmentation}

The proposed face segmentation method selects from the data only the face and neck regions, which are the areas with useful information for the morphological analysis. The typical objects removed as part of this process include: the shoulders, some hair or objects in the background. The method is based on the 3D face pose estimation utilizing a generic 3D face model.

Each depth frame is transformed into a 3D point cloud using the intrinsic parameters of the sensor. Then, based on the face pose estimation described above, a 3D generic face model (see Figure 1) is transformed to match the input 3D data. The initial rotation matrix is defined by the three estimated pose rotation angles and the translation vector contains the estimated coordinates of the head center.

Subsequently all the points belonging to the model are transformed by using the rigid registration process between the model and the input $3 \mathrm{D}$ data using iterative closest point (ICP) [40]. After the registration, all the points belonging to the model are projected into a $2 \mathrm{D}$ image using the camera calibration parameters building a 2D sparse mask (see Figure 2). In order to generate a dense and continuous mask, standard mathematical morphology techniques are applied to the mask image (erosion and dilation, see implementations in [41]). 


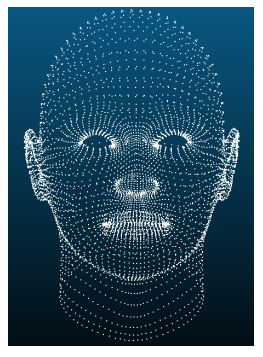

Figure 1: Generic 3D face model used for segmentation, created using the software FaceGen (www.facegen.com)
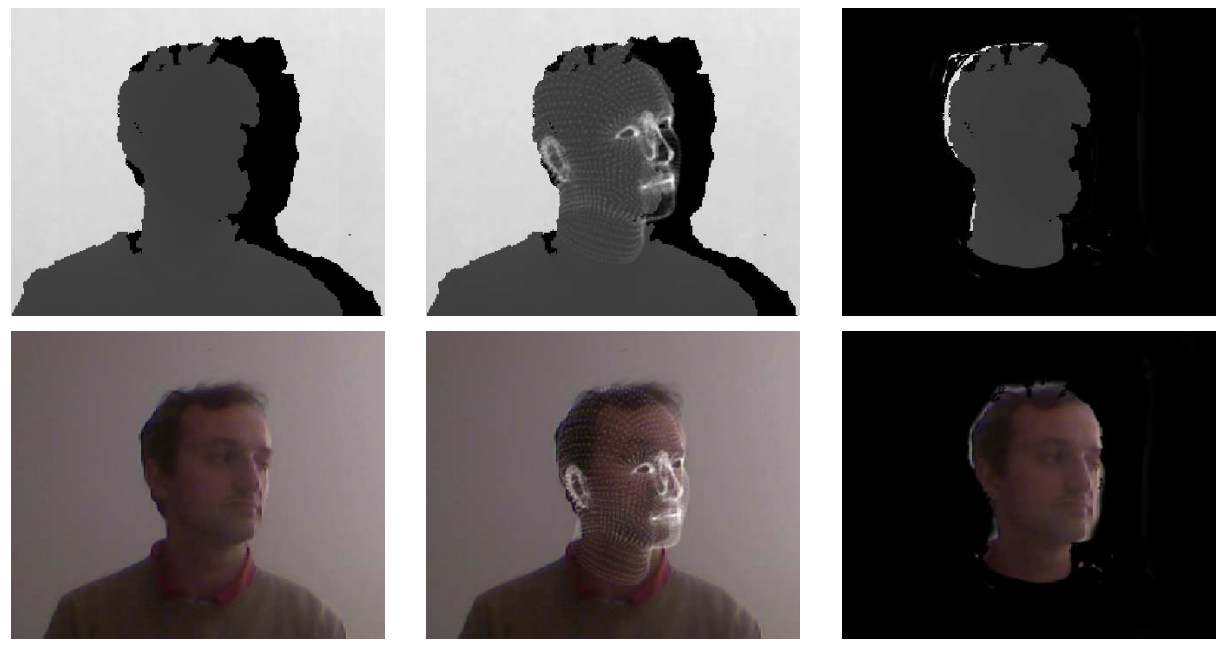

Figure 2: First row: Input depth frame from the sensor (left), rigidly registered generic face model projected onto the depth frame (centre), segmented face after applying the proposed method (right). Second row: Colour images, shown only for better visualization of the processing pipeline. The colour information is not used in the proposed system. 


\subsubsection{Registration of range scans}

The $3 \mathrm{D}$ reconstruction process is based on merging the $3 \mathrm{D}$ data from captured frames to reconstruct the scene. The proposed system uses the methods described in [36] and its implementation available in [40]. That implementation was extended for this work, to include the techniques used for face segmentation explained in Section 3.1.2.

The original reconstruction method proposed in [36] uses a moving sensor to capture different points of view in order to reconstructs static scenes. In this work, a new functionality has been added to allow reconstructing a face when the sensor is in a fixed position and the person is moving. Using the segmentation proposed in Section 3.1.2, the person motion is reversed to estimate the relative motion of the sensor with the head being in a virtual fixed position. Such approach enables unobtrusive measurements with very limited user interaction in the framework of the proposed Wize Mirror developed as part of the SEMEOTICONS project. The original procedure of moving the sensor to reconstruct the user face can be still used with the proposed method, as the only data used for reconstruction are the results of segmentation, if the person is still and the sensor is moving, the movement will be equivalent to fixing the sensor and rotating the head. Such approach may be useful when subject cannot move or stand still.

For each new 3D input the points are tracked by aligning a surface measurement $\left(V_{k}, N_{k}\right)$ (vertex and normal vectors maps) against the model prediction from the previous frame $\left(\hat{V}_{k-1}, \hat{N}_{k-1}\right)$.

\subsubsection{Surface reconstruction}

In order to perform the surface reconstruction, a volumetric truncated signed distance function (TSDF) is used (see [36]). After the registration process, the $3 \mathrm{D}$ points are fused into a $3 \mathrm{D}$ global face reconstruction. The global reconstructed volume is formed by the weighted average of all individual TSDFs computed for each depth map. This fusion computes the denoised global TSDF from multiple noisy TSDF measurements.

The reconstructed meshes obtained using the method described in [36] are of good quality, however, they do not fully meet the requirements for a correct morphological analysis of the 3D face reconstructions. The mesh has to be a manifold and contain no holes and no duplicated points or triangles.

Therefore the Poisson surface estimation method, described in [37], is applied on the 3D point cloud extracted from the TSDF in order to obtain a new mesh compatible with the requirements imposed by the morphological 

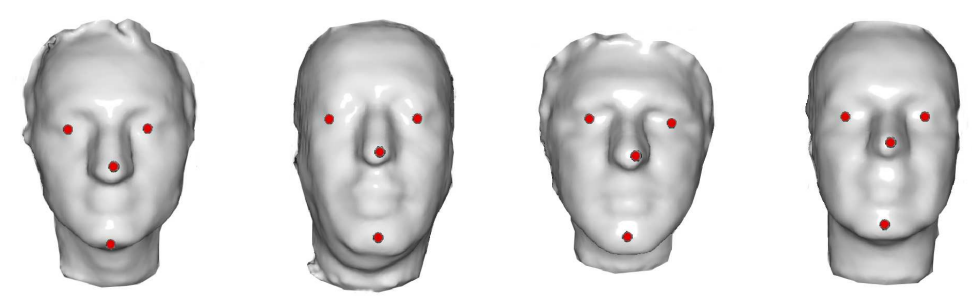

Figure 3: Example of 3D reconstruction and labelling results using the proposed method. Rendered using the Phong's shader provided in Meshlab [42]

analysis. In the reported here results, the open source Point Cloud Library (PCL, [40]) implementation of the Poisson meshing was used. Representative examples of the surface reconstruction are shown in Figure 3.

\subsubsection{Labelling}

The labelling process produces as output the approximate positions of the centre of the eyes, tip of the nose and centre of the chin on the reconstructed mesh. This labelling is an important requirement for the subsequent morphological analysis. The method proposed to tackle this problem is based on a deformable model. In that method the model is labelled manually only once, marking the points of interest. Then the model is registered to the reconstructed face and after the deformable fitting, the labels end up close to the relevant anatomical landmarks.

The detailed description of the adopted deformable model is provided in [43]. A low dimensional shape space vector (SSV) of the statistical shape model (SSM) is used to represent 3D faces. The SSM has been calculated using a model-based surface registration process. Two different stages are needed in this process: model building and hierarchical model fitting. The point distribution model described in [44] is used for building the SSM. First, the point correspondences between faces in the training database are estimated. Subsequently, based on the estimated point correspondences, the statistical shape model is built using PCA. As result a new face $Q$ can be approximately represented by the linear model $Q=W b+\bar{Q}$, where $\bar{Q}$, where $\bar{Q}$ is a mean face, $W=\left[u_{1}, \ldots, u_{k}\right]$ is the shape matrix of $\mathrm{k}$ eigen-faces $\left[u_{1}, \ldots, u_{k}\right]$ and $b=\left[b_{1}, \ldots, b_{k}\right]$ is the shape space vector (SSV) which controls the contribution of each eigen-face in the approximated face $Q$. The hierarchical model fitting algorithm estimates iteratively and in turn the 
shape (vector $b$ ) and the pose (face position and orientation).

The automatic labelling results were compared with the ground truth labels manually selected on 3D facial reconstructions of 22 test subjects. These manual annotations were not aided by textural information as only 3D shape data were used in that process. The average error of the automatic landmark positioning was estimated at $5.6 \mathrm{~mm}, 4.3 \mathrm{~mm}$ and $6.9 \mathrm{~mm}$ for the eyes, nose and chin respectively. These should be compared with the corresponding estimated ground truth annotation errors of $2.5 \mathrm{~mm}, 1.5 \mathrm{~mm}$ and $2.2 \mathrm{~mm}$ respectively. The ground truth was calculated as the average from three manual annotations performed by two observers. The estimated eye landmarks positions are used to estimate the interpupillary distance, subsequently used for normalisation of the proposed face measurements (see Section 3.2). The normalised eye labelling error [45] (defined as $\max (\mathrm{eL}, \mathrm{eR}) / \mathrm{d}$, with $\mathrm{eL} / \mathrm{eR}$ representing distance between the estimated and ground truth position of the left/right eye and d denoting the ground truth interpupillary distance) was estimated at approximately 0.1. As reported in [46] this is comparable with the error obtained by the other methods used for eye detection. The average interpupillary distance measurement error is $5.2 \mathrm{~mm}$ with the estimated corresponding ground truth annotation error of $2.3 \mathrm{~mm}$. It should be noted that normalisation using the interpupillary distance only partially corrects for differences between subjects faces as it accounts only for an overall variability of the face size but it does not take into account inherent differences in face morphology between subjects. The results reported in Section 5, show agreement between measurements based on manually labelled high resolution 3D facial scans using commercial Artec scanner and those computed based on the proposed automatically labelled 3D facial reconstructions. Therefore it can be concluded that the proposed facial features (see Section 3.2) are not overly sensitive to the accuracy of the labels/landmarks detection.

\subsubsection{D reconstruction validation}

The 3D face reconstruction method has been validated through different experiments using a plastic head model (see Figure 4) and real faces from subjects during different acquisition campaigns (see Figure 3). In order to check the stability of the 3D model obtained using the proposed reconstruction method, the reconstructions of the plastic head model were repeated multiple times with differently acquired range data. For the experiment presented in Figure 4, the plastic head model was scanned five times, from slightly different positions and inclinations in front of the sensor. Four differ- 


\begin{tabular}{|c|c|c|}
\hline Number of points & Mean distance $(\mathrm{mm})$ & Std. deviation $(\mathrm{mm})$ \\
\hline 62295 & 0.5 & 2.2 \\
\hline 60777 & 0.5 & 1.5 \\
\hline 63798 & 0.4 & 2.5 \\
\hline 67851 & 0.2 & 2.9 \\
\hline $\mathbf{6 3 6 8 0}$ & $\mathbf{0 . 4}$ & $\mathbf{2 . 2}$ \\
\hline
\end{tabular}

Table 1: 3D reconstruction statistics: number of points per reconstruction, mean distance between those points and the reference reconstruction and standard deviation of the distances. Each row corresponds to one reconstruction presented in Figure 4 and the last row shows the average.

ent reconstructions were compared to the randomly selected reference reconstruction. Each reconstruction was registered to the reference one using the ICP algorithm provided in the CloudCompare software [47]. Afterwards the average distance and the standard deviation were estimated using the cloud to mesh tool available in [47], and finally the distance map is obtained using the calculated distances by the same software.

As it can be seen in Table 1, the average error is only $0.4 \mathrm{~mm}$, and the standard deviation $2.2 \mathrm{~mm}$, which indicates that the scanner provides repeatable and consistent reconstructions of the same face surface independently from the changes in the face position or orientation. This is an important result as it shows that the random reconstruction error (from the sensor and registration process), which is difficult to correct, is very small. Therefore, the obtained reconstructions are suitable for the subsequent morphological analysis.

\section{2. $3 D$ face features}

This work aims to define reliable and automatic measures of face morphology which are correlated with weight and fat-related parameters, and which can be automatically computed on the reconstructed 3D faces.

As observed in Section 2.2, most of the methods in the literature deal with 3D scanned bodies rather than faces. Also, the methods dealing with faces are based on 2D images rather than 3D data. Nevertheless, faces are inherently three-dimensional objects. Thanks to the availability of complete $3 \mathrm{D}$ data rather than just images, it is possible to take advantage of modern 3D shape analysis techniques, such as the computation of geodesic distances 

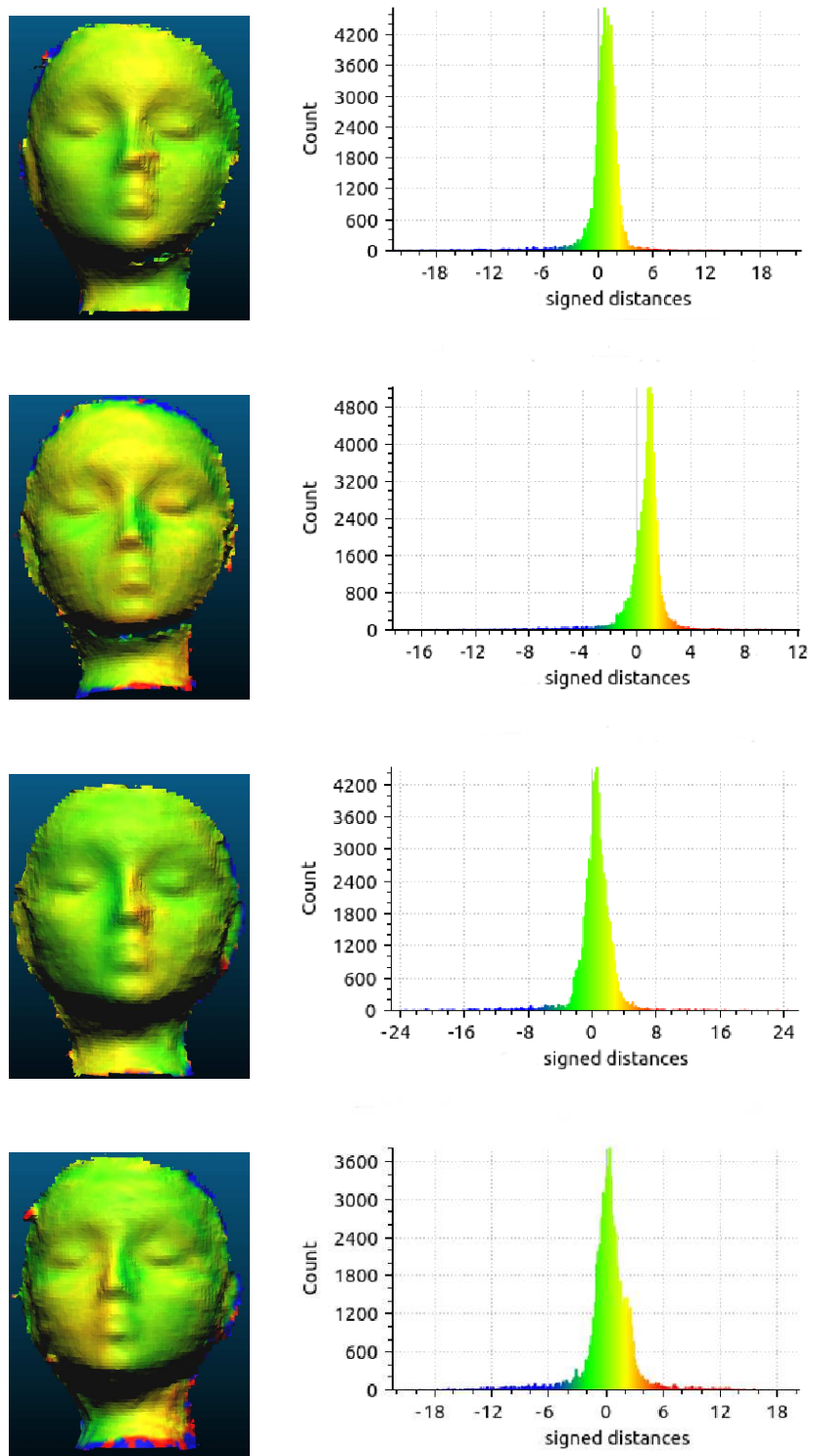

Figure 4: Comparison between 3D reconstructions obtained using the proposed method. On the left, signed distance maps between the current and the reference reconstructions. On the right, histograms calculated with the number of points belonging to the reconstructed face and clustered depending on their signed distance (in millimetres) to the reference face. 
on the surface, thus using surface feature to consider the whole geometry of the face.

Moreover, most of the methods in the literature are based on the accurate location of anatomical landmarks on the face [34, 48, 30]. Nevertheless, locating landmarks with optimal accuracy on 3D acquired data could be difficult, especially for poorly geometrically characterized landmarks. Since this study aims to devise measures which can be computed automatically on 3D data acquired via a low-cost scanner, so as to be part of a self-monitoring automatic system, the proposed method uses shape measures which are independent of the precise, optimal location of a large set of anatomical landmarks. Indeed they require the detection of only three landmarks (namely the eyes and the nose tip, visualised also in Figure 3), automatically located on 3D face meshes as explained in Section 3.1.5: the nose tip is used for the location of the geometric objects involved in the shape measurements, while the distance between eyes is used for normalization, partially accounting for differences among subjects. Additionally, another measure is proposed: the length of a geodesic path through the neck region, defined thanks to the detection of the chin landmark (see Figure 3). Thus, the considered shape measures result objective, normalized, and easily computable from scanned data.

The process of putting on weight is likely to involve complex shape changes. Figure 5 shows a simulation based on a synthetic model, the Basel Face Model (BFM). The BFM is a morphable face model built from the registered 3D scans of 100 male and 100 female subjects [49]. By applying principal component analysis to the registered scans, the regression coefficients, accounting for the main face shape variations according to gender, age, height and weight, are extracted. By controlling the weight parameters, one can simulate a 10-step fattening process on a randomly generated seed face, as shown in Figure 5 (left). Figure 5 (right) shows the comparison between the fattest face and the seed one; the colours represent the pointto-point distances between the seed and the morphed face, with red (blue) indicating the regions most (least) affected by the weight changes. It can be seen that most of the changes affect the peripheral regions of the face, and the neck region. These are the regions which are analysed by the proposed descriptors as detailed in the next sections.

Even though the facial measurements are not fully normalised with respect to the differences among subjects, in the perspective of the integration into the Wize Mirror, such a normalization is not strongly required, because the self-monitoring is based on their variation over time on a single subject, 
Figure 5: A synthetic face fattening in ten steps (left); Point-to-point distances between the fat face and the normal one, mapped onto the fat face in false colors, values increasing from blue to red (right).

rather than on their absolute value.

The first measure proposed is Morpho $_{E}$. Morpho ${ }_{E}$ is the maximum length among the curves $c_{i}$ given by the intersection of the face mesh with a set of Euclidean spheres centered on the nose tip and radius varying in the range $\left[0, R_{E}\right]$ (Figure 6 , left). The choice of the radius $R_{E}$ is crucial to satisfy a set of possibly conflicting requirements. First, $R_{E}$ has to be sufficiently large to guarantee that the curves span a sufficiently large and informative face area. Second, $R_{E}$ must be sufficiently small not to reach critical face regions, namely those which are most likely to be affected from occlusions (e.g., due to hair) and therefore $3 \mathrm{D}$ reconstruction errors. Finally, it would be convenient to have $R_{E}$ defined automatically for each subject. In Section 5, we experiment with $R_{E}=k_{E} * D_{\text {eye }}, D_{\text {eye }}$ being the distance between the subject's eyes and $k_{E}$ set to 1.5 , which empirically proved to be a reasonable value. The range $\left[0, R_{E}\right]$ is sampled into 20 equally spaced intervals. Then,

$$
\operatorname{Morpho}_{E}=\max _{i=1, \ldots, 20}\left\{\text { length }\left(c_{i}\right)\right\} \text {. }
$$

A check is performed on the reconstructed 3D mesh to ensure that the biggest sphere does not intersect the mesh boundary, guaranteeing that no errors are introduced due to occlusions and reconstruction errors.

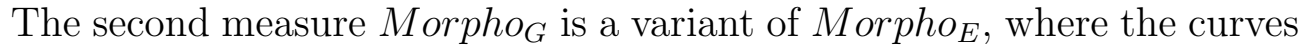
on the mesh are defined as the boundaries of a set of geodesic disks centered in the nose tip. A geodesic disk of radius $r$ and center $C$ is the locus of points having geodesic distance $r$ from $C$; briefly speaking, geodesic distances measure the shortest path between two points along the surface, that is, the path one would follow if bounded to walk on the surface of the object [26]. Therefore, geodesic distances capture information which is substantially different from Euclidean distances. We compute geodesic distances using the the Dijkstra algorithm, which takes $O(n \log (n))$ [50]. As above, the sphere radii vary in the range $\left[0, R_{G}\right]$. In the experiments performed, $R_{G}=k_{G} * D_{\text {eye }}$, with 

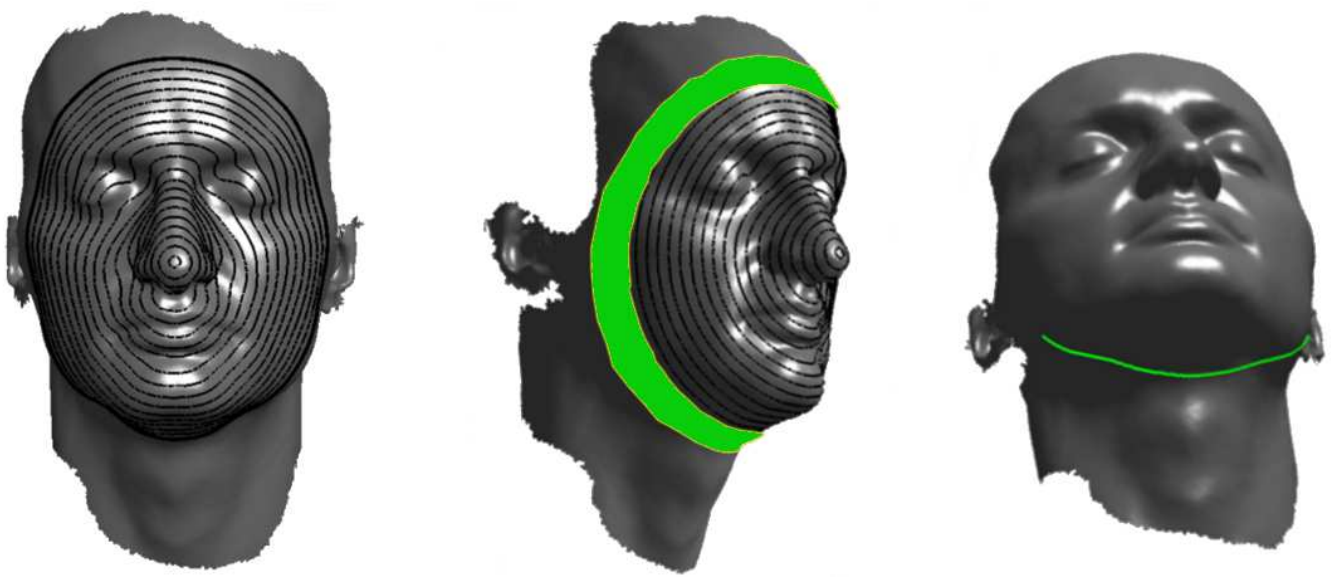

Figure 6: LEFT. Morpho : $_{\text {: }}$ in bold, the curve which realizes the maximum length among the intersection between the face and the set of spheres centered in the nose tip. MIDDLE. Annuli $_{1.4,1.55}$ measures the area of the green region. RIGHT. $A B_{1.7,1.4}$ measures the length of the green geodesic path.

$k_{G}$ set to 1.65 . Again, the range $\left[0, R_{G}\right]$ is sampled into 20 equally-spaced intervals.

The third measure Annuli $_{R_{1}, R_{2}}$ is defined as the area of the annular region of the mesh enclosed between two Euclidean spheres centered in the nose tip and having radii $R_{1} * D_{\text {eye }}$ and $R_{2} * D_{\text {eye }}$ respectively, with $R_{1}=k_{1} * D_{\text {eye }}$ and $R_{2}=k_{2} * D_{\text {eye }}, k_{1}=1.4$ and $k_{2}=1.55$. Whereas Morpho $o_{E}$ and Morpho $_{G}$ are length measurements, Annuli $R_{1}, R_{2}$ is a surface measure; for a visualization see Figure 6, middle.

The last measure proposed, $A B_{\lambda_{1}, \lambda_{2}}$, focuses on the neck region, and on the region below the chin in particular. Indeed, $A B_{\lambda_{1}, \lambda_{2}}$ is defined as the length of the geodesic path joining two feature points on the face mesh. The two points are given by the intersection of two spheres: the first is centered in the nose tip, and has radius $\lambda_{1} * D_{\text {eye }}$, the latter is centered in the chin, and has radius $\lambda_{2} * D_{\text {eye }}$. Here $\lambda_{1}=\lambda_{2}=1.6$, values found empirically to be reasonable for the whole set of data, defining points below the inferior earlobes (see Figure 6, right). In the experiments performed the geodesic joining the two points always went through the neck region, under the chin.

The measurements defined above are:

- well-defined, easy to implement, and computationally efficient;

- independent of rotation and translation (thanks to the use of spheres), 
and scale (thanks to the normalization with respect to the distance between the eyes);

- robust against noise and pose estimation errors.

\section{Experimental setting}

In order to show the robustness of the four digital measures we defined, and evaluate the correlation of these measures with the standard indexes of cardio-metabolic risk and weight, we have performed some experiment on a set of 30 volunteers, 13 women and 17 men, aged in-between 29 and 62 years. 22 (6 women, 16 men) were enrolled in an acquisition campaign held at the CNR premises in Pisa (Italy) in May, 2015; and other 8 (7 women, 1 man) were enrolled in October, 2015, in the same location. The study was approved by the Ethical Committee of the "Azienda Ospedaliera Universitaria Pisana", protocol n.213/2014 approved on September 25 ${ }^{\text {th }}, 2014$; all patients provided a signed informed consent before enrollment.

\subsection{Standard anthropometric measurements}

The following physical parameters were collected by the medical staff for each volunteer: gender, age, height, weight, neck circumference, waist circumference, hip circumference. Then, BMI was calculated. The waist circumference was measured at the umbilicus, and the hip circumference in a horizontal plane at the level of the maximal extension of the buttocks. Additional parameters related to body composition were collected: fat-free mass (FFM) was measured by electrical bioimpedance (scale manufactured by Tanita, Japan), and fat mass (FM) was calculated as the difference between body weight and FFM. Table 2 shows the characteristics of the studied population: mean, standard deviation, and the range of variation for each characteristic as min - max values.

\subsection{Digital anthropometric measurements}

The first 22 subjects had their face scanned, both with a low-cost scanner based on the Kinect range sensor, and with a commercial portable structured light scanner (ARTEC Eva). The aim was to assess how much the mesh quality affects the results. The other 8 subjects were scanned only with the Kinect. 


\begin{tabular}{|c|c|c|c|c|c|c|c|}
\hline & $\begin{array}{c}\text { Weight } \\
(\mathrm{kg})\end{array}$ & $\begin{array}{c}\text { BMI } \\
\left(\mathrm{kg} / \mathrm{m}^{2}\right)\end{array}$ & $\begin{array}{c}\text { WC } \\
(\mathrm{cm})\end{array}$ & $\begin{array}{c}\text { HC } \\
(\mathrm{cm})\end{array}$ & $\begin{array}{c}\text { NC } \\
(\mathrm{cm})\end{array}$ & $\begin{array}{c}\text { FFM } \\
(\%)\end{array}$ & $\begin{array}{c}\text { FM } \\
(\%)\end{array}$ \\
\hline mean & 73.36 & 25.06 & 89.04 & 100.25 & 35.77 & 73.08 & 26.92 \\
\hline$\sigma$ & 21.37 & 5.88 & 17.91 & 14.37 & 3.75 & 9.19 & 9.19 \\
\hline range & $48.9-168$ & $17.8-51.8$ & $66-170$ & $83-165$ & $30-46$ & $51-91.5$ & $8.5-49$ \\
\hline
\end{tabular}

Table 2: Characteristics of the studied population, 13 women and 17 men. (BMI: body mass index; WC: waist circumference; HC: hip circumference; NC: neck circumference; FFM: fat-free mass; FM: fat mass; $\sigma$ : standard deviation)

There are differences between the acquisition protocol used for capturing with the depth sensor and the commercial scanner. While the depth sensor was fixed in a position and the user simply rotated the head left and right, the ARTEC Eva was moved around the head of the subject in order to scan the face. The proposed acquisition protocol for the low-cost reconstruction system requires only the interaction of the user during approximately five seconds. The system informs the user that a slow rotation of the head is needed, first to the left and then to the right. While the user is rotating the head, the depth frames are being saved and they are used afterwards to perform the 3D reconstruction following the work-flow described in Section 3.1. When using the ARTEC Eva, the user remained still and an operator moved the scanner around the user's head, resulting in a longer scanning time. However, due to different scanning techniques used for both sensors, the structured light scanner provides reconstructions with higher resolution, which is also associated to a much higher cost.

\subsection{Statistical Analysis}

The Kolmogorov-Smirnov tests [51, pp. 392-394] were applied to data, and they resulted all normally distributed; hence, parametric tests were used to compare the mean among groups. In Section 5 the concordance between data coming from different hardware was assessed computing the intra-class correlation coefficient (ICC, [52]); the Pearson's correlation coefficient was used to evaluate the existing relations between facial features and physical parameters.

Structural Equation Modeling (SEM) is a combination of factor analysis and multiple regression: a methodology to estimate and test a network of relations among observed and latent variables [53] and, in the current setting, to evaluate whether the facial features may be condensed into a single 


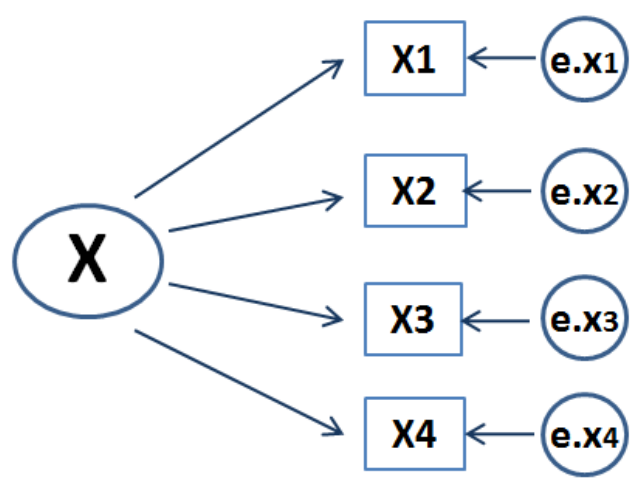

Figure 7: An example path diagram of a Structure Equation Model: $x 1, \ldots, x 4$ are the observed variables; $X$ is the latent variable; e. $x i$ is the error of $x i$.

indicator. A sample of a structural equation model is given by the following equation system, also illustrated through a path diagram in Figure 7 . The path coefficients $\beta_{i}$ in the equation system define how much each observed variable contributes to the latent variable.

$$
\left\{\begin{array}{l}
x 1=\alpha 1+\beta 1 \cdot X+\text { e.x } 1 \\
x 2=\alpha 2+\beta 2 \cdot X+e . x 2 \\
x 3=\alpha 3+\beta 3 \cdot X+\text { e.x } 3 \\
x 4=\alpha 4+\beta 4 \cdot X+\text { e.x } 4
\end{array}\right.
$$

The quality of a statistical model is assessed measuring its fit, through tests or indicators (see [54]). Here, the SEM model is evaluated with two indexes: (i) Standardized Root Mean Square Residual (SRMR); (ii) Comparative Fit Index (CFI). The SRMR is a measure of the mean absolute value of the covariance residuals and it is defined as the difference between the observed correlation and the predicted correlation. A model is considered to show a good fit if the SRMR is $\leq 0.10$. The Comparative Fit Index (CFI) is equal to the discrepancy function adjusted for sample size; CFI ranges from 0 to 1 and a model with CFI values $\geq 0.95$ is considered to have an acceptable fit.

Statistical analysis was conducted using SPSS Statistic 19.0 program and STATA 13.0 software. 


\section{Results}

First of all, it was verified that the use of a commercial or a low-cost scanner did not affect significantly the results, via assessing the concordance between the features coming from the Artec scans, and from the Kinect scans. Interrater agreement between the two sets of data was assessed with the single score ICC. The interpretation of the ICC values, according [52], is: 0-0.2 poor agreement; $0.3-0.4$, fair agreement; $0.5-0.6$, moderate agreement; $0.7-0.8$, strong agreement; $>0.8$, almost perfect agreement. Interrater agreement between measures from Artec and Kinect resulted very high: strong to almost perfect for all items; the correlation was moderate $(\mathrm{ICC}=.678$, $95 \% \mathrm{CIs}=.225-.866$ ) only between measures $A B_{\lambda_{1}, \lambda_{2}}$ (see Table 3). These

\begin{tabular}{|c|c|c|c|c|}
\hline & Morpho $_{E}$ & Morpho $_{G}$ & Annuli $_{R_{1}, R_{2}}$ & $A B_{\lambda_{1}, \lambda_{2}}$ \\
\hline ICC & .913 & .894 & .775 & .678 \\
\hline 95\%-CIs & $.790-.964$ & $.745-.956$ & $.458-.907$ & $.225-.866$ \\
\hline
\end{tabular}

Table 3: Interrater agreement between data acquired with Kinect and Artec, expressed as intra-class correlation coefficient. ICC: Intraclass Correlation Coefficient; CIs: Confidence interval.

values revealed that the geometric features selected do not strongly depend on mesh resolution and accuracy. In order to better appreciate the measurements' robustness against the precise location of landmarks and the mesh resolution, a visualization of each measurement, computed on the two sets of 3D facial data, is provided in Figure 8.

Hence, on the basis of the statistical methods described above, in the following subsections there is a description of the results, supporting the relationship between the proposed computational descriptors and the physical parameters: weight, BMI, WC, HC, NC, FM. The FM (fat mass) was added in order to get an index of body composition. First the discussion relates to each single facial feature, then to the composite index Face Morphology, built as the latent variable of a single-factor model.

\subsection{Correlations between face features and physical parameters}

All facial features are highly correlated with weight, BMI and NC, up to $r=0.78$, and all are highly significant, with a $p$-value $\leq 0.001$ for all features. Morpho $o_{E}$ and Morpho $_{G}$ are also highly correlated with WC and 

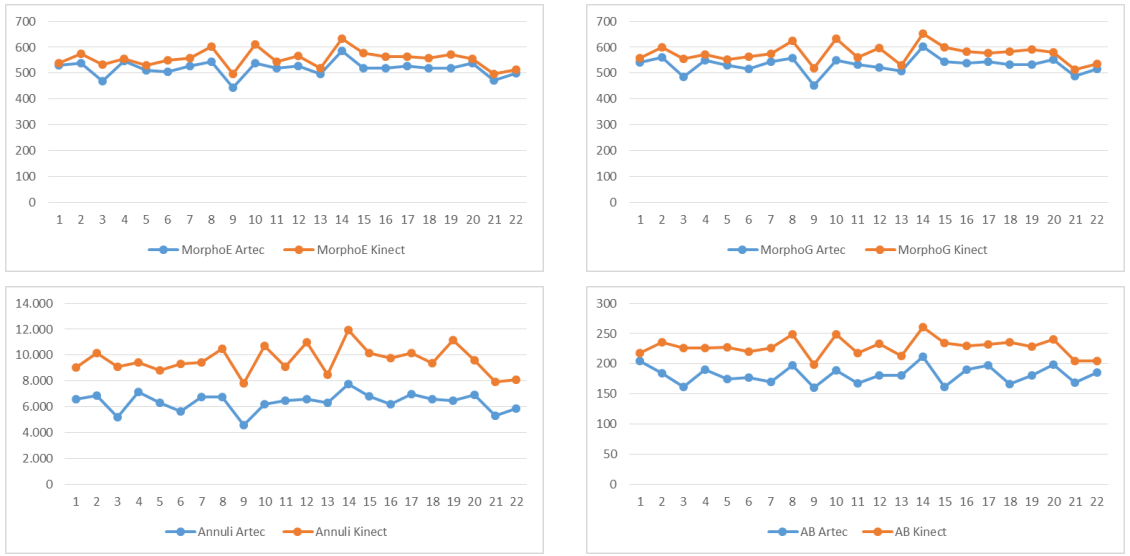

Figure 8: Four graphs showing the concordance between the measurements computed on the 3D facial data acquired with the Artec scan and manually labelled, and those acquired with the low-cost sensor and automatically labelled.
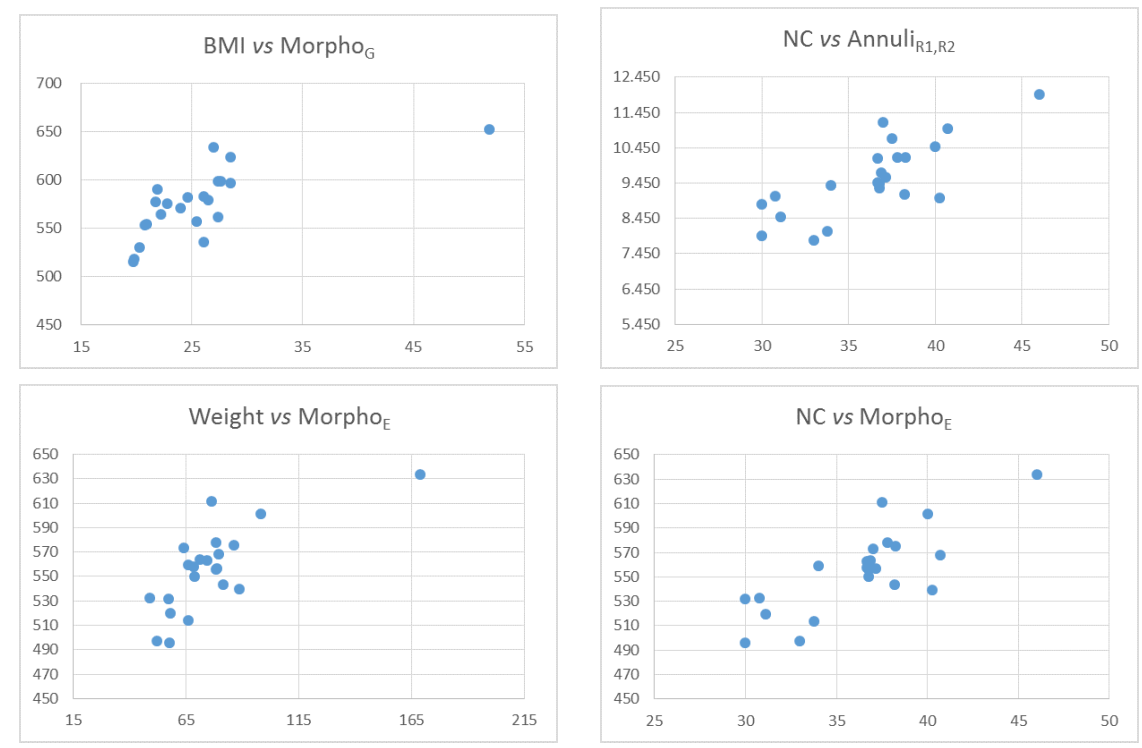

Figure 9: Some plots showing the correlation between the physical parameters related to body fat and the proposed measurements: BMI and Morpho $_{G}$, neck circumference and Annuli $_{R 1, R 2}$, weight and Morpho $_{E}$, and neck circumference and Morpho $E$. 
HC, up to $r=0.619$, and with high significance $(p \leq 0.001)$. The correlation between the features Annuli $_{R_{1}, R_{2}}, A B_{\lambda_{1}, \lambda_{2}}$ and the parameters WC and $\mathrm{HC}$ is a bit lower, but still significant, except for Annuli $_{R_{1}, R_{2}}$ and WC. Some of these correlations are shown in the charts in Figure 9, while Table 4 shows the Pearson's correlation coefficients between computational 3D face features and all the considered fat-related physical parameters. Given the relatively small size of the sample and the fact that the data are not specific to any range of body weight and fat, but include subjects in diverse fitness conditions, the results are very good, in that they show strong correlation between automatically extracted 3D facial features and fat-related physical parameters.

Concerning FM, the facial features show a moderate positive correlation, yet the $p$-values show that the correlation is not significant. This might be due to the sample size, and also to the fact that the sample includes both men and women, and gender significantly affects FM values; the use of a corrective factor is currently under study.

\begin{tabular}{|cc|c|c|c|c|}
\hline & & Morpho $_{E}$ & Morpho $_{G}$ & Annuli $_{R_{1}, R_{2}}$ & AB $_{\lambda_{1}, \lambda_{2}}$ \\
\hline Weight & $r$ & .733 & .719 & .669 & .675 \\
& p-value & .000 & .000 & .001 & .001 \\
\hline BMI & $r$ & .711 & .716 & .651 & .671 \\
& p-value & .000 & .000 & .001 & .001 \\
\hline WC & $r$ & .614 & .619 & .547 & .579 \\
& p-value & .002 & .002 & .08 & .005 \\
\hline HC & $r$ & .569 & .568 & .518 & .557 \\
& p-value & .007 & .007 & .016 & .009 \\
\hline NC & $r$ & .788 & .781 & .778 & .648 \\
& p-value & .000 & .000 & .000 & .001 \\
\hline FM & $r$ & .272 & .316 & .211 & .349 \\
& p-value & .221 & .152 & .347 & .112 \\
\hline
\end{tabular}

Table 4: Pearson's correlation coefficients between facial features and weight and fatrelated indexes (BMI: body mass index; WC: waist circumference; HC: hip circumference; NC: neck circumference; FM: fat mass) 


\subsection{SEM analysis}

A single-factor model is built on the set of facial features extracted from the Kinect scans (observed variables): Morpho, Morpho $_{G}$, Annuli $_{R_{1}, R_{2}}$, $A B_{\lambda_{1}, \lambda_{2}}$; the latent variable is Face Morphology. The goodness of fit of the Face Morphology model, in the studied population, results high: $\chi^{2}=137.45$, $\mathrm{p}$-value $=0.0000 ; \mathrm{SRMR}=0.008 ; \mathrm{CFI}=0.953$. Then the SEM model is correctly built. Figure 10 shows the relations between the latent variable Face

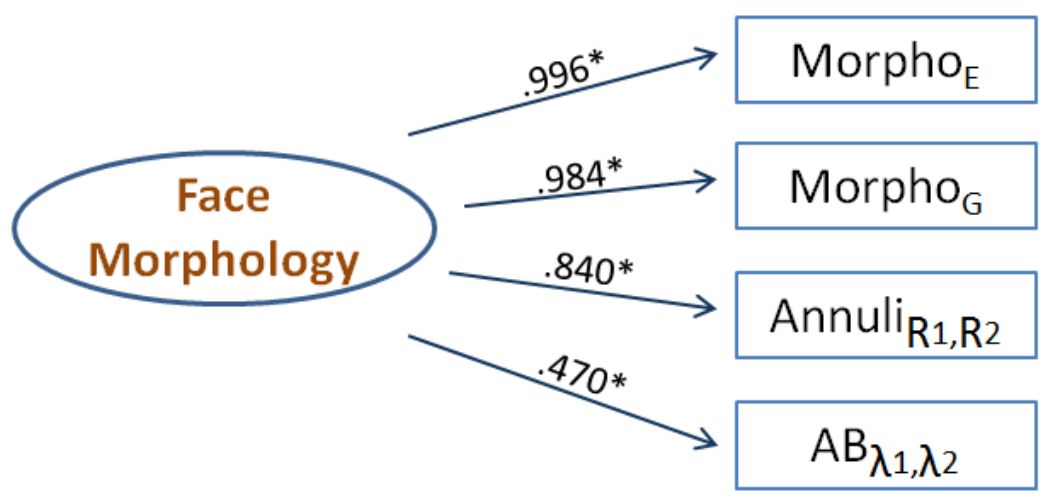

Figure 10: Path diagram of the single-factor model, with path coefficients labeling each arrow. The path coefficients are standardized: values $<0.10$ indicate a small effect; values around 0.30 indicate a medium effect; and values $>0.50$ indicate a large effect. (* means that $\mathrm{p}$-value $<0.0001$ )

Morphology and the facial features, quantified by the labels on the arrows. All the standardized coefficients are above .84, with the exception of $A B_{\lambda_{1}, \lambda_{2}}$; thus, Face Morphology provides a very good explanation of the observed variables.

The mean vector and variance matrix of the model were used to compute the factor score of the Face Morphology model, via a linear regression. Table 5 reports the Pearson's correlation analysis between weight-related indexes and the factor score of the structural equation model: the factor score correlates better than each single facial feature with all physical parameters (but FM). 


\begin{tabular}{|c|c|c|c|c|c|c|}
\hline & Weight & BMI & WC & HC & NC & FM \\
\hline$r$ & .742 & .724 & .633 & .580 & .804 & .300 \\
\hline
\end{tabular}

Table 5: Pearson's $r$ between weight-related indexes and factor score of the model (BMI: body mass index; WC: waist circumference; HC: hip circumference; NC: neck circumference; FM: fat mass). All coefficients have p-values $<0.0001$.

\section{Discussion and Conclusion}

We presented an automatic system able to reconstruct a 3D face and extract measurements, which can be automatically computed and do not require neither high scanning resolution nor pose adjustments. The results on the sample of the studied subjects, without any known pathology or medical treatment, allow us to formulate two conjectures: (i) a relevant relation exists among face morphology and body weight and fat-related parameters, including markers of central obesity, an important component of cardio-metabolic risk; (ii) the relation is stable in the general adult population.

Looking back to the existing literature dealing with face morphology, cited in Section 2.2, it is appropriate to make a few comments. Banabilh et al. in [13] carry out an accurate analysis of the face shape, using the 3D stereophotogrammetry, in order to detect patients more prone to severe form of sleep apnea; hence, although this study is one of the first investigating the relations between $3 \mathrm{D}$ accurate facial data and specific healthy/unhealthy condition, it cannot be properly compared to our study, nor with respect to the methodology neither to the objective. Other works (such as by Coetzee et al. [8] and Tinlin et al. [9]) aim to assess the health status focusing on body fat; on the contrary, they measure the perceived fat adiposity, hence they do not provide a reproducible measure for the assessment of the health status. Ferrario et al. [30] consider the excess body fat in obese adolescents and the facial features studied are computed using 50landmarks manually located for each subject; while the measurements here proposed use only 4 facial landmarks, automatically labelled; also the target population is different.

In $[32,33]$ the study population is divided into four groups (by age and gender) and the waist circumference is used to classify each subject into two classes: normal and obese, defined with respect to the visceral obesity, assessed through the waist circumference. Hence, the predictive potential of the facial geometric features considered is evaluate in [33] by computing the AUC (area under the receiver operating characteristic curve) and the 
Cohen's Kappa, and such analysis shows that for each group there exist facial descriptors which are good in discriminating the two classes. Comparing to the present work, the features considered in [33] are computed on 2D data collected manually by expert technicians, whilst the proposed descriptors are based on 3D data, automatically acquired and labelled; also, as described in the previous Section, in spite of the small size of the sample considered, the Pearson's correlations result moderate to strong between the 3D automatic descriptors and a set of body fat related parameters (NC, weight, BMI, WC). The statistical analysis is carried out considering such 3D descriptors as continuous variables and the prediction potential of the proposed descriptors (and of the composite index) has not been assessed; on the other hand, the correlation patterns found indicate that such descriptors may be used in the future to classify the subjects into more than two classes, with respect to body fat.

The obtained results encourage us to increase the number of enrolled subjects, also taking into consideration gender differences, and to prolong the observation for each subject. The main objectives are: (i) to better clarify, and possibly quantify, the link between facial descriptors and factors of cardio-metabolic risk; (ii) to define normal ranges and cutoff values with respect to the cardio-metabolic risk through well-established risk charts (HEART SCORE, Fatty-Liver index, HOMA index, FINRISK); (iii) understand whether these descriptors could be used to properly follow the temporal variations of the risk factors related to body weight and fat.

Our findings may be considered as a first step in the development of interactive, non obtrusive monitoring systems, based on low-cost depth sensors, able to provide reliable estimates of body weight and related parameters.

\section{Acknowledgments}

This work has been partially supported by the European Community's Seventh Framework Programme (FP7/2013-2016) under the grant agreement n. 611516 (SEMEOTICONS - SEMEiotic Oriented Technology for Individuals CardiOmetabolic risk self-assessmeNt and Self-monitoring). The authors would also thank dr. G. Coppini, and dr. A. Gastaldelli for many valuable discussions. 


\section{References}

[1] Obesity and overweight: fact sheet n 311; 2015, http://www. who.int/ mediacentre/factsheets/fs311/en/.

[2] J. D. Douketis, J. W. Feightner, J. Attia, W. F. Feldman, Periodic health examination, 1999 update: 1. detection, prevention and treatment of obesity. canadian task force on preventive health care, Canadian Medical Association Journal 160 (4) (1999) 513-525.

[3] Global health observatory data, http://www.who.int/gho/ncd/risk _factors/overweight/en/.

[4] E. B. Loucks, Z. Schuman-Olivier, W. B. Britton, D. M. Fresco, G. Desbordes, J. A. Brewer, C. Fulwiler, Mindfulness and cardiovascular disease risk: state of the evidence, plausible mechanisms, and theoretical framework, Current Cardiology Reports 17 (12), doi:10.1007/s11886015-0668-7.

[5] Semeoticons fp7-ict-2013-10 european project (2013), http://www . semeoticons.eu/.

[6] S. Colantonio, G. Coppini, D. Germanese, D. Giorgi, M. Magrini, P. Marraccini, M. Martinelli, M.-A. Morales, M. A. Pascali, G. Raccichini, M. Righi, O. Salvetti, A smart mirror to promote a healthy lifestyle, Biosystems Engineering, Innovations in Medicine and Healthcare 138 (2015) 33-43, doi:10.1016/j.biosystemseng.2015.06.008.

[7] Y. Andreu-Cabedo, P. Henriquez, S. Colantonio, G. Coppini, R. Favilla, D. Germanese, G. Giannakakis, D. Giorgi, M. Larsson, P. Marraccini, M. Martinelli, B. J. Matuszewski, M. Milanic, M. A. Pascali, M. Pediaditis, G. Raccichini, L. Randeberg, O. Salvetti, T. Stromberg, Mirror mirror on the wall... an intelligent multisensory mirror for well-being self-assessment, in: Multimedia and Expo (ICME), 2015 IEEE International Conference on, 2015, pp. 1-6. doi:10.1109/ICME.2015.7177468.

[8] V. Coetzee, D. I. Perrett, I. D. Stephen, Facial adiposity: A cue to health?, Perception 38 (2009) 1700-1711. doi:10.1068/p6423.

[9] R. M. Tinlin, C. D. Watkins, L. L. M. Welling, L. M. DeBruine, E. A. S. Al-Dujaili, B. C. Jones, Perceived facial adiposity conveys information 
about women's health, British Journal of Psychology 104 (2013) 235248.

[10] G. Coppini, R. Favilla, A. Gastaldelli, S. Colantonio, P. Marraccini, Moving medical semeiotics to the digital realm. semeoticons approach to face signs of cardiometabolic risk, in: SUPERHEAL 2014 HEALTHINF, 2014, pp. 606-613.

[11] J. Wang, D. Gallagher, J. C. Thornton, W. Yu, M. Horlick, F. X. PiSunyer, Validation of a 3-dimensional photonic scanner for the measurement of body volumes, dimensions and percentage body fat., American Journal of Clinical Nutrition (2006) 809-816.

[12] J. C. Wells, T. J. Cole, D. Bruner, P. Treleaven, Body shape in american and british adults: between-country and inter-ethnic comparisons., International Journal of Obesity 32 (1) (2008) 152-159.

[13] S. M. Banabilh, A. H. Suzina, S. Dinsuhaimi, A. R. Samsudin, G. D. Singh, Craniofacial obesity in patients with obstructive sleep apnea, Sleep and Breathing 13 (2009) 19-24. doi:10.1007/s11325-008-0211-9.

[14] S. R. Millar, I. J. Perry, C. M. Phillips, Surrogate measures of adiposity and cardiometabolic risk - why the uncertainty? a review of recent meta-analytic studies, Journal of Diabetes and Metabolism S11:004. doi:10.4172/2155-6156.S11-004.

[15] A. Keys, F. Fidanza, M. J. Karvonen, N. Kimura, H. L. Taylor, Indices of relative weight and obesity, Journal of Chronic Diseases 25 (1972) 329-343.

[16] C. D. Brown, M. Higgins, K. A. Donato, F. C. Rohde, R. Garrison, E. Obarzanek, N. D. Ernst, M. Horan, Body mass index and the prevalence of hypertension and dyslipidaemia, Obesity Research 8 (2000) 605619, doi:10.1038/oby2000.79.

[17] P. Bjorntorp, Visceral obesity: a civilization syndrome, Obesity Research 1 (1993) 206-222.

[18] D. R. Brenner, K. Tepylo, K. M. Eny, L. E. Cahill, A. El-Sohemy, Comparison of body mass index and waist circumference as predictors of 
cardiometabolic health in a population of young canadian adults, Diabetology \& Metabolic Syndrome 2 (1) (2010) 1-8, doi:10.1186/1758$5996-2-28$.

[19] I. Janssen, P. T. Katzmarzyk, R. Ross, Body mass index, waist circumference, and health risk: evidence in support of current national institutes of health guidelines, Archives of Internal Medicine 162 (18) (2002) 2074-2079.

[20] A. D. Liese, et al., Development of the multiple metabolic syndrome in the aric cohort: joint contribution of insulin, bmi, and whr. atherosclerosis risk in communities, Ann Epidemiol 7 (6) (1997) 407-416.

[21] B. Heitmann, et al., Hip circumference and cardiovascular morbidity and mortality in men and women, Obesity Research 12 (2004) 482-487.

[22] R. Huxley, S. Mendis, E. Zheleznyakov, S. Reddy, J. Chan, Body mass index, waist circumference and waist:hip ratio as predictors of cardiovascular risk-a review of the literature, European Journal of Clinical Nutrition 1 (2010) 16-22, doi:10.1038/ejcn.2009.68.

[23] A. Onat, G. Hergenc, H. Yuksel, G. Can, E. Ayhan, Z. Kaya, et al., Neck circumference as a measure of central obesity: associations with metabolic syndrome and obstructive sleep apnea syndrome beyond waist circumference, Clinical Nutrition 28 (1) (2009) 46-51.

[24] C. Stabe, A. C. Vasques, M. M. Lima, M. A. Tambascia, J. C. Pareja, A. Yamanaka, et al., Neck circumference as a simple tool for identifying the metabolic syndrome and insulin resistance: results from the brazilian metabolic syndrome study, Clinical Endocrinology (Oxf) 78 (6) (2013) 874-881.

[25] L. Ben-Noun, A. Laor, Relationship between changes in neck circumference and cardiovascular risk factors, Experimental \& Clinical Cardiology 11 (1) (2006) 14-20.

[26] S. Biasotti, B. Falcidieno, D. Giorgi, M. Spagnuolo, Mathematical tools for shape analysis and description, Synthesis Lectures on Computer Graphics and Animation 6 (2) (2014) 1-138. 
[27] C. Velardo, J.-L. Dugelay, Weight estimation from visual body appearance, in: BTAS 2010, 4th IEEE International Conference on Biometrics: Theory, Applications and Systems, September 27-29, 2010, Washington DC, USA, 2010, pp. 1-6.

[28] C. Velardo, J.-L. Dugelay, M. Paleari, P. Ariano, Building the space scale or how to weight a person with no gravity, in: ESPA 2012, IEEE 1st International Conference on Emerging Signal Processing Applications, January 12-14, 2012, Las Vegas, USA, 2012. doi:10.1109/ESPA.2012.6152447.

[29] A. Giachetti, C. Lovato, F. Piscitelli, C. Milanese, C. Zancanaro, Robust automatic measurement of $3 \mathrm{~d}$ scanned models for human body fat estimation., IEEE Journal of Biomedical and Health Informatics 19 (2) (2015) 660-667.

[30] V. F. Ferrario, C. Dellavia, G. M. Tartaglia, M. Turci, C. Sforza, Softtissue facial morphology in obese adolescents: a three-dimensional non invasive assessment, The Angle Orthodontist 74 (1) (2004) 37-42.

[31] J. Djordjevic, D. A. Lawlor, A. L. Zhurov, et al., A population-based cross-sectional study of the association between facial morphology and cardiometabolic risk factors in adolescence, in: BMJ Open, Vol. 3, 2013, pii: e002910.

[32] B. J. Lee, J. H. Do, J. K. Kim, A classification method of normal and overweight females based on facial features for automated medical applications, Journal of Biomedicine and Biotechnology (2012:834578). doi:10.1155/2012/834578.

[33] B. J. Lee, J. K. Kim, Predicting visceral obesity based on facial characteristics, BMC Complementary and Alternative Medicine 14 (2014). doi:10.1186/1472-6882-14-248.

[34] D. Giorgi, M. A. Pascali, G. Raccichini, S. Colantonio, O. Salvetti, Morphological analysis of $3 \mathrm{~d}$ faces for weight gain assessment, in: Proc. of EG 3DOR 2015, Eurographics 2015 Workshop on 3D Object Retrieval, Zurich (Switzerland) - May 2-3, 2015, 2015, pp. 55-62.

[35] L. G. Farkas, Anthropometry of the head and face, Raven Press, New York, 1994. 
[36] S. Izadi, O. Hilliges, D. Molyneaux, D. Kim, A. J. Davison, P. Kohli, J. Shotton, S. Hodges, A. Fitzgibbon, R. A. Newcombe, Kinectfusion: Real-time dense surface mapping and tracking, in: International Symposium on Mixed and Augmented Reality, 2011, pp. 127-136.

[37] M. Kazhdan, M. Bolitho, H. Hoppe, Poisson surface reconstruction, in: Eurographics symposium on geometry processing, 2006, pp. 61-70.

[38] P. Henriquez, O. Higuera, B. J. Matuszewski, Head pose tracking for immersive applications, in: Proceedings of IEEE International Conference on Image Processing 2014, 2014, pp. 1957 - 1961.

[39] G. Fanelli, T. Weise, J. Gall, L. V. Gool, Real time head pose estimation from consumer depth cameras, in: Annual Symposium of the German Association for Pattern Recognition, Vol. 6835, 2011, pp. 101-110.

[40] R. Rusu, S. Cousins, 3d is here: Point cloud library (pcl), in: IEEE International Conference on Robotics and Automation (ICRA), Shanghai, China, 2011, pp. 1-4.

[41] Itseez, The OpenCV Reference Manual, 2nd Edition, [See http:// opencv.org/] (April 2014).

[42] Visual Computing Lab - ISTI - CNR, Meshlab, 1st Edition, [Retrieved from http://meshlab. sourceforge.net] (October 2015).

[43] W. Quan, B. J. Matuszewski, L.-K. Shark, Improved 3-d facial representation through statistical shape model, in: IEEE International Conference on Image Processing, 2010, pp. 2433-2436.

[44] D. H. Cooper, T. F. Cootes, C. J. Taylor, J. Graham, Active shape models - their training and application, Computer Vision and Image Understanding 61 (1995) 38-59.

[45] J. O., K. K., F. R., Robust face detection using the hausdorff distance, in: Proc. AVBPA 2001, LNCS, Springer, 2001, pp. 90-95.

[46] F. Timm, E. Barth, Accurate eye centre localisation by means of gradients, in: Proc. VISAPP 2011, Vol. 11, 2011, pp. 125-130. 
[47] EDF RD, Telecom ParisTech, CloudCompare (version 2.6) [GPL software], 2nd Edition, [Retrieved from http://www.cloudcompare.org/] (October 2015).

[48] F. L. Bookstein, Biometrics, biomathematics and the morphometric synthesis, Bulletin of mathematical biology 58 (2) (1996) 313-365.

[49] P. Paysan, R. Knothe, B. Amberg, S. Romdhani, T. Vetter, A 3d face model for pose and illumination invariant face recognition, in: IEEE Proc. of the 6th IEEE International Conference on Advanced Video and Signal based Surveillance (AVSS) for Security, Safety and Monitoring in Smart Environments, 2009, pp. 296 - 301.

[50] T. H. Cormen, C. E. Leiserson, R. L.Rivest, Introduction to algorithms., The MIT Press, Cambridge, MA, 1994.

[51] I. M. Chakravarti, R. G. Laha, J. Roy, Handbook of Methods of Applied Statistics, Vol. I, John Wiley and Sons, 1967.

[52] C. A. E. Nickerson, A note on a concordance correlation coefficient to evaluate reproducibility, Biometrics (International Biometric Society) 53 (4) (1997) 1503-1507. doi:10.2307/2533516.

[53] R. H. Hoyle, The structural equation modeling approach: Basic concepts and fundamental issues, Thousand Oaks, CA: Sage Publications, Inc., 1995.

[54] D. Hooper, J. Coughlan, M. R. Mullen, Structural equation modelling: Guidelines for determining model fit, Journal of Business Research Methods 6 (2008) 53-60. 